Article

\title{
Broadband Ultra-Deep Sub-Diffraction-Limit Optical Focusing by Metallic Graded-Index (MGRIN) Lenses
}

\author{
Yechuan Zhu ${ }^{1,2}$, Weizheng Yuan ${ }^{1,2}$, Hao Sun ${ }^{1,2}$ and Yiting $\mathrm{Yu}^{1,2, *}$ \\ 1 Key Laboratory of Micro/Nano Systems for Aerospace, Ministry of Education, Xi'an 710072, China; \\ yechuanzhu_30@hotmail.de (Y.Z.); yuanwz@nwpu.edu.cn (W.Y.); sunhao0878@outlook.com (H.S.) \\ 2 Key Laboratory of Micro- and Nano-Electro-Mechanical Systems of Shaanxi Province, \\ Northwestern Polytechnical University, Xi'an 710072, China \\ * Correspondence: yyt@nwpu.edu.cn
}

Received: 20 July 2017; Accepted: 9 August 2017; Published: 12 August 2017

\begin{abstract}
The development of techniques for efficiently confining energy in the visible and infrared spectral regions to the deep subwavelength spatial scale with dimensions as small as a few nanometers would have great significance for scientific research and engineering practices. Such an ability to manipulate light is impossible for conventional dielectric lenses due to the diffraction limit. Here, we propose a metallic graded-index (MGRIN) lens formed by an array of coupled metallic waveguides with identical nanoscale widths embedded by index-varying dielectrics to enable the optical nanofocusing. The focusing mechanism of the MGRIN lens is theoretically investigated based on Hamiltonian optics, which are verified by the finite-difference time-domain (FDTD) method. Numerical results reveal that an ultra-deep subwavelength focus of $8 \mathrm{~nm}(\lambda / 500)$ with a long focal depth $(1.93 \lambda)$ and enhanced field intensity can be achieved. Moreover, the nanofocusing capability of the MGRIN lens without redesigning the structure can be well kept when the incident wavelength changes over a broad range from visible to infrared. Our design of optical nanofocusing shows great potential for use in nano-optics and nanotechnology.
\end{abstract}

Keywords: nanofocusing; coupled metallic waveguides; metallic graded-index lens; Hamiltonian optics

\section{Introduction}

The spatial resolution of conventional optical devices is restricted by the diffraction to nearly half the operating wavelength, which greatly limits the performance of all the imaging and focusing systems that lie at the heart of modern biology, electronics, and optical integrated circuits [1-3]. In the past two decades, a great number of plasmonic devices have been designed to exceed the diffraction limit and manipulate the properties of light with nanometer-scale precision based on surface plasmon polaritons (SPPs) [4-7]. In particular, many efforts have been made in nanofocusing of light using various metallic structures [8], such as plasmonic nanoantennas and tapered metallic structures.

Plasmonic nanoantennas function as optical resonators that can support highly localized plasmon modes, thus leading to the strong enhancement of the localized field near the antennas [9]. Unlike nanofocusing through localized surface plasmon resonance, plasmonic nanofocusing by tapered metallic structures involves an increasing concentration of light energy into a nanoscale spatial region as the SPP mode propagates along a tapered plasmonic waveguide with gradually varying parameters [8]. The metallic structures for such nanofocusing mainly include tapered metal rods with rounded tips [10-15], metal wedges surrounded by dielectrics [16-18], tapered metal strips on dielectric substrates [19-21], tapered gaps formed by metal-insulator-metal (MIM) structures [22-26], and metallic V-grooves [27]. These open great opportunities for the development of a new class 
of nano-optical devices and techniques, such as optical signal processing in highly integrated nanophotonic circuits [28], nano-optical sensors [29], nanoimaging [30], and nanomechanics [31].

Completely different from the two typical ways of optical nanofocusing mentioned above, in this work, we propose a unique method for nanofocusing using a planar metallic graded-index (MGRIN) lens that is composed of multilayer coupled MIM waveguides. Numerical simulation confirms our design and demonstrates that nanofocusing with a long focal depth and enhanced field intensity can be achieved with the MGRIN lens for broadband frequencies from visible to infrared. This planar structure can find applications in wavelength division multiplexing, nanolithography, and highly integrated optical circuits.

\section{MGRIN Lens Design}

Metallic waveguides have an extremely distinctive advantage over dielectric structures in their support for deep subwavelength modes [32], thus enabling the manipulation of light at the nanoscale. Additionally, metallic waveguide arrays have been extensively proposed for optical focusing [33-38]. Particularly, in 2009, Verslegers et al. [39] designed an aperiodic metallic waveguide array using Hamiltonian optics and demonstrated a deep-subwavelength focus of $30 \mathrm{~nm}$, as small as $\lambda / 100$ ( $\lambda$ being the operating wavelength), in the central waveguide. However, in their design, the waveguide widths must increase from the two sides to the center, which limits the further reduction of the central waveguide width and the resulting nanofocusing capability. In contrast, an array of coupled metallic waveguides of the constant width and gold spacing but filled with different dielectrics is employed to form our MGRIN lens for the nanofocusing scheme, as shown in Figure 1.

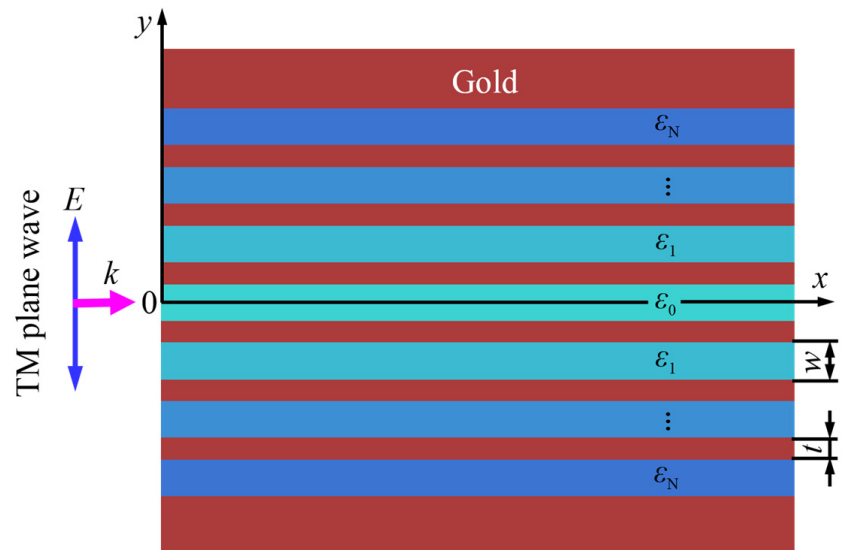

Figure 1. The schematic of a metallic graded-index (MGRIN) lens formed by coupled waveguides of uniform width and gold spacing under the normal incidence of a transverse magnetic plane wave. The structure is symmetric with respect to the central waveguide at $y=0$, and $\varepsilon_{n}(0 \leq n \leq \mathrm{N})(n$ the integer with the values of $0,1,2 \ldots \mathrm{N}$ ) represents the permittivity of the dielectric in the waveguide $n$.

In our design, the structure is symmetric with respect to the central waveguide at $y=0$ and semi-infinite in the $z$ direction, which is normally illuminated by a transverse magnetic (TM) plane wave. Because the permittivities of the dielectrics do not vary much between one waveguide and its adjacent counterpart, this structure can be locally considered periodic. Therefore, the consequent dispersion equation for a waveguide can be derived using the transfer matrix method [40]:

$$
\cos \left[k_{y}(w+t)\right]=\cos \left(k_{1} w\right) \cos \left(k_{2} t\right)-\frac{\varepsilon_{m}^{2} k_{1}^{2}+\varepsilon_{d}^{2} k_{2}^{2}}{2 \varepsilon_{m} \varepsilon_{d} k_{1} k_{2}} \sin \left(k_{1} w\right) \sin \left(k_{2} t\right)
$$

with $k_{1}=\left(\varepsilon_{d} k_{0}^{2}-k_{x}^{2}\right)^{1 / 2}$ and $k_{2}=\left(\varepsilon_{m} k_{0}^{2}-k_{x}^{2}\right)^{1 / 2}$, where $w$ is the waveguide width, $t$ is the metal spacing between two adjacent waveguides, $k_{0}$ is the free space wave vector, and $k_{x}$ is the propagation 
wave vector in the $x$ direction. $\varepsilon_{m}$ and $\varepsilon_{d}$ are the permittivities of the metal and dielectric material in the waveguide, respectively. Our nanofocusing structure is built based on the following propagation constant profile of the metallic waveguides along the $y$ axis:

$$
\beta_{s}(y)=\beta_{s, 0}\left(1+a y^{2}\right)
$$

where $\beta_{s}$ is the symmetric solution of $k_{x}$ in Equation (1) for $k_{y}=0$, and $\beta_{s, 0}$ is the corresponding value of the central waveguide. $a$ is the gradient parameter. Since the effective refractive index of a waveguide is $n_{e}=\beta_{s} / k_{0}$, Equation (2) can be transformed into the following:

$$
n_{e}(y)=n_{e, 0}\left(1+a y^{2}\right)
$$

This quadratic effective index profile is similar to the design of a conventional graded-index lens [41], albeit the effective index increasing from the center to the side due to the negative refraction of a metallic waveguide array [42]. This is the reason why we consider this metallic waveguide array as an MGRIN lens.

On the other hand, by choosing a proper parameter $b$, we can obtain the following approximation:

$$
\beta_{a}(y)=\beta_{a, 0}\left(1+b y^{2}\right)
$$

where $\beta_{a}$ is the antisymmetric solution of $k_{x}$ in Equation (1) for $k_{y}=\pi /(w+t)$, and $\beta_{a, 0}$ is the corresponding solution of the central waveguide. From Equations (2) and (4), the Hamiltonian can be deduced:

$$
H\left(y, k_{y}\right)=k_{x}-\beta_{s, 0}-a \beta_{s, 0} y^{2}+\frac{1}{4}\left(\beta_{s, 0}-\beta_{a, 0}\right)(w+t)^{2} k_{y}^{2}
$$

Furthermore, the trajectory of a light ray in the structure can be solved by:

$$
\frac{d y}{d x}=\frac{\partial H\left(y, k_{y}\right)}{\partial k_{y}}, \frac{d k_{y}}{d x}=-\frac{\partial H\left(y, k_{y}\right)}{\partial y}
$$

From Equations (5) and (6), the analytical solution of a ray trajectory can be derived as follows:

$$
y(x)=C_{1} \sin (G x)+C_{2} \cos (G x)
$$

with $G=\left[\left(\beta_{a, 0}-\beta_{s, 0}\right) a \beta_{s, 0}\right]^{1 / 2}(w+t), C_{1}$ and $C_{2}$ are constants related to the position and angle of the incident ray. Assuming that at $x=0$, the position is $y=y_{0}$ and the corresponding slope is $y^{\prime}=y_{0}{ }^{\prime}$, the above equation can be transformed into:

$$
y(x)=\frac{1}{G} y_{0}^{\prime} \sin (G x)+y_{0} \cos (G x)
$$

In the case of normal incidence, since $y_{0}{ }^{\prime}=0$, a ray trajectory can be further written as:

$$
y(x)=y_{0} \cos (G x)
$$

From Equation (9), it can be concluded that for a normally incident plane wave, all rays go through a cosine trajectory and consequently intersect at a focal length of $\pi /(2 G)$ when they propagate in the structure of an MGRIN lens. This is the self-focusing mechanism of our MGRIN lens.

\section{Results and Discussion}

To demonstrate the focusing behavior, an MGRIN lens operating at a wavelength of $1 \mu \mathrm{m}$ was designed with $\varepsilon_{0}=1$ and $\varepsilon_{\mathrm{N}}=1.69$. At this wavelength, the permittivity of gold is $\varepsilon_{m}=-40.764+1.261 i$ [43]. We considered a structure with a total of 51 waveguides that have the 
same width of $10 \mathrm{~nm}$ and are uniformly separated by $30 \mathrm{~nm}$ of gold. The required permittivity of the dielectric in the $n$th waveguide $(0 \leq n \leq 25)$ is calculated by using Equations (1) and (2), as shown in Figure 2. The maximum variation in the dielectric constant between the adjacent waveguides is less than 0.06 . Thus, it is reasonable to consider the structure to be locally periodic.

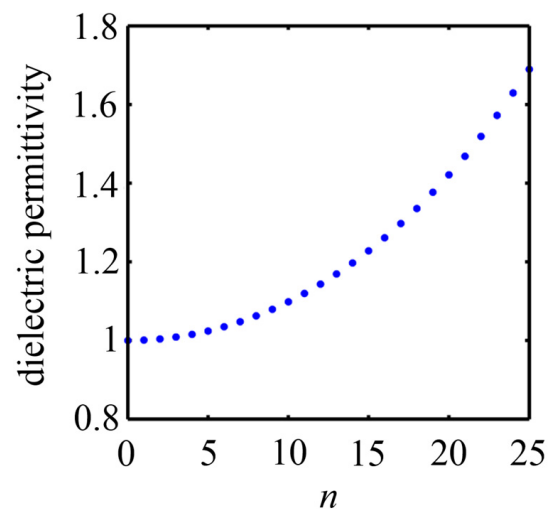

Figure 2. The required permittivity of the dielectric in the $n$th waveguide of an MGRIN lens working at $\lambda=1 \mu \mathrm{m}$. The MGRIN lens comprises a total of 51 waveguides and the dielectric constant increases from 1 at the center to 1.69 at the sides.

By using the finite-difference time-domain (FDTD) simulation, the focusing performance of the designed MGRIN lens is analyzed. In simulations, the grid size is set to $1 \mathrm{~nm}$ in both $x$ and $y$ directions to model the fine features of the electromagnetic field in the structure. Perfectly matched layers as the absorbing boundary conditions are applied around the computational domain. The incident TM-polarized plane wave is defined by setting the electric field component $E_{y}$ with the amplitude of 1 .

Figure 3a presents the simulated electric field intensity distribution of the structure, which clearly confirms the focusing behavior of the MGRIN lens. The realized full width at half maximum (FWHM) at the focus is $8 \mathrm{~nm}(\lambda / 125)$, demonstrating the ultra-deep sub-diffraction-limit focusing. This is 12.5 times smaller than the FWHM of $100 \mathrm{~nm}$ achieved in the previous study, using a structure with varying waveguide widths [39] for the same operating wavelength. The focal depth (DOF) is $1.24 \mu \mathrm{m}$ (Figure $3 \mathrm{~b}$ ), and more than one wavelength, which is difficult to implement by using the previous nanofocusing approaches. Furthermore, the simulated focal length is $6.44 \mu \mathrm{m}$, which is close to the theoretical result of $6.87 \mu \mathrm{m}$ calculated by the Hamiltonian optics (i.e., $\pi /(2 G)$ ). Meanwhile, compared with the incident plane wave, the field intensity at the focus is enhanced to 92.
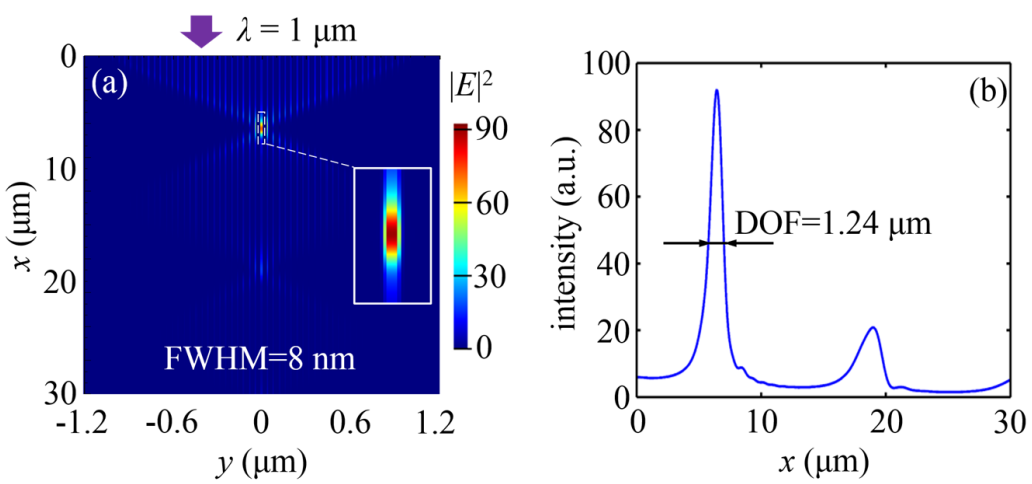

Figure 3. Ultra-deep sub-diffraction-limit focusing of an MGRIN lens. (a) FDTD-simulated electric field intensity pattern. The inset shows the enlarged view for the electric intensity distribution of the focus; (b) The derived $|E|^{2}$ on the optical axis. The FWHM and focal depth of the focus are $8 \mathrm{~nm}$ and $1.24 \mu \mathrm{m}$, respectively. 
In the previous research [39], the focusing structure needed to be redesigned when operating at a longer wavelength. In contrast, this is unnecessary for our design. We can use the same structure to obtain the nanofocusing effect for a wide range of wavelengths. Figure $4 \mathrm{a}-\mathrm{c}$ show the simulated electric field intensity patterns for longer wavelengths of $2-4 \mu \mathrm{m}$ (at $2 \mu \mathrm{m}, \varepsilon_{m}=-183.23+7.522 i$; at $3 \mu \mathrm{m}, \varepsilon_{m}=-415.98+22.462 i$; at $\left.4 \mu \mathrm{m}, \varepsilon_{m}=-747.36+51.625 i\right)$ with the same structure as the one designed for the wavelength $\lambda=1 \mu \mathrm{m}$. These simulation results illustrate the similar focusing behavior. For the shorter wavelengths in the visible range down to $650 \mathrm{~nm}$, focusing can also be realized, as shown in Figure 5. Nevertheless, for shorter wavelengths, the nanofocusing effect cannot be observed in the structure due to the losses near the cutoff frequencies for plasma oscillations [44]. Besides the operating wavelength, the propagation losses in the structure also depend on the spacing between metallic waveguides. Losses increase with the metallic spacing. However, the metallic spacing cannot be too small to provide the capability for subwavelength optical confinement. Therefore, the metallic spacing should be appropriately selected for the nanofocusing scheme.
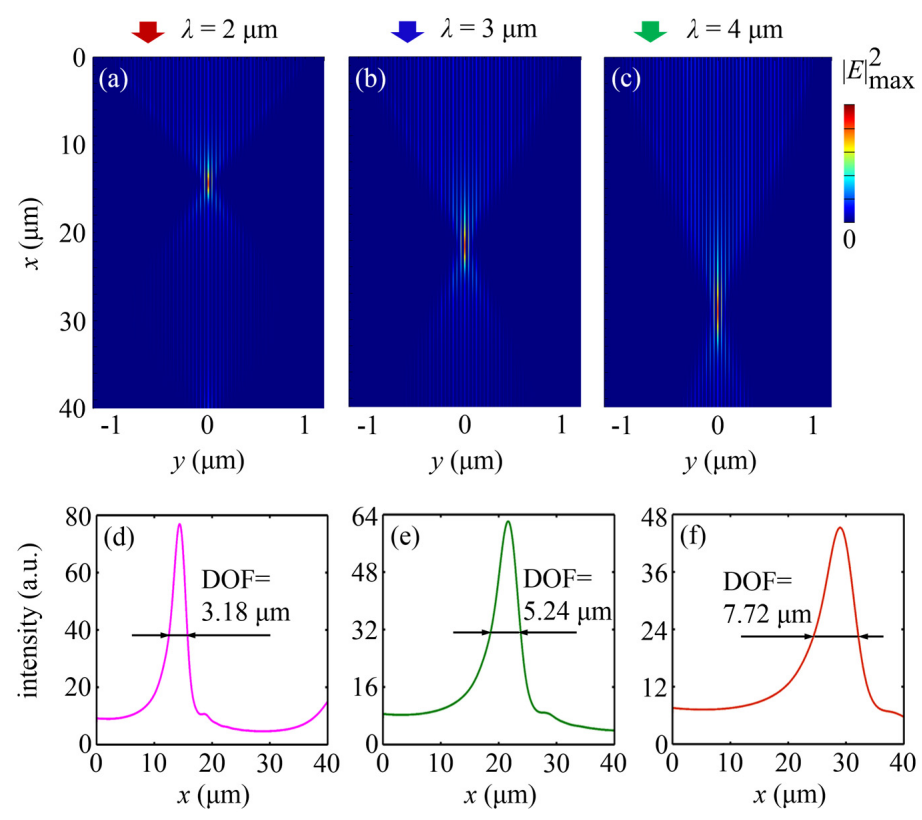

Figure 4. Ultra-deep sub-diffraction-limit focusing of an MGRIN lens working at longer wavelengths. $(\mathbf{a}-\mathbf{c})$ FDTD-simulated electric field intensity patterns for the wavelengths of $2-4 \mu \mathrm{m}$, respectively; $(\mathbf{d}-\mathbf{f})$ The corresponding $|E|^{2}$ on the optical axis. The FWHMs of the three foci are all $8 \mathrm{~nm}$. The focal depths at $2-4 \mu \mathrm{m}$ are $3.18 \mu \mathrm{m}, 5.24 \mu \mathrm{m}$, and $7.72 \mu \mathrm{m}$, respectively.

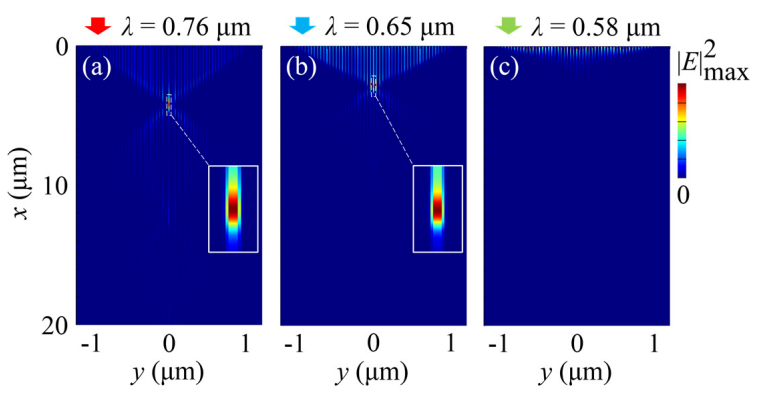

Figure 5. Ultra-deep sub-diffraction-limit focusing of an MGRIN lens working at the shorter wavelengths. (a-c) FDTD-simulated electric field intensity patterns for the wavelengths of $0.76 \mu \mathrm{m}, 0.65 \mu \mathrm{m}$ and $0.58 \mu \mathrm{m}$, respectively. The permittivity of gold at these three wavelengths is $-20.273+0.703 i,-12.266+0.779 i$, and $-7.571+1.141 i$, respectively. The FWHMs of the two foci at $0.76 \mu \mathrm{m}$ and $0.65 \mu \mathrm{m}$ are both $8 \mathrm{~nm}$. The corresponding focal depths are $0.86 \mu \mathrm{m}$ and $0.88 \mu \mathrm{m}$, respectively. 
Remarkably, the FWHMs of the foci considered are all $8 \mathrm{~nm}$, far beyond the diffraction limit. For the operating wavelength of $4 \mu \mathrm{m}$, a focus as small as $\lambda / 500 \mathrm{can}$ be obtained. In addition, when the incident wavelength varies from $0.65 \mu \mathrm{m}$ to $4 \mu \mathrm{m}$, the focal length of the MGRIN lens increases from $2.88 \mu \mathrm{m}$ to $28.98 \mu \mathrm{m}$ accordingly (displayed in Figure 6a). Therefore, the focal length of an MGRIN lens can be modulated by controlling the working wavelength, apart from tuning the design parameters based on Equation (2). The focal depth also increases with the wavelength (depicted in Figure 6b), which is larger than one operating wavelength. Furthermore, the field intensity enhancement for all the foci is higher than 20, as shown in Figure 6c.
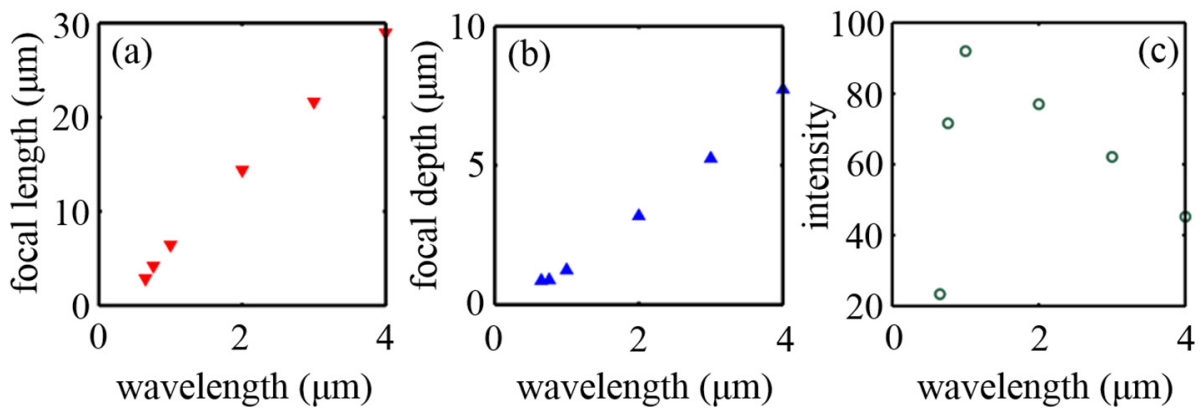

Figure 6. The focusing performance of an MGRIN lens working at various wavelengths from $0.65 \mu \mathrm{m}$ to $4 \mu \mathrm{m}$. (a-c) Focal length, focal depth, and the maximum intensity at the focus varying as a function of the incident wavelength.

The physical mechanism behind the broadband focusing of an MGRIN lens is the small change in the effective index of a metallic waveguide. For instance, when the incident wavelength varies from $1 \mu \mathrm{m}$ to $4 \mu \mathrm{m}$, the effective index of the waveguide at the side changes from 2.63 to 2.49 . As for the central waveguide, the effective index change is much smaller, as shown in Figure 7. As a result, the effective indices of waveguides comprising the MGRIN lens are approximate, to meet Equation (3). This is the reason for the realization of nanofocusing with only one structure for a broad range of wavelengths.

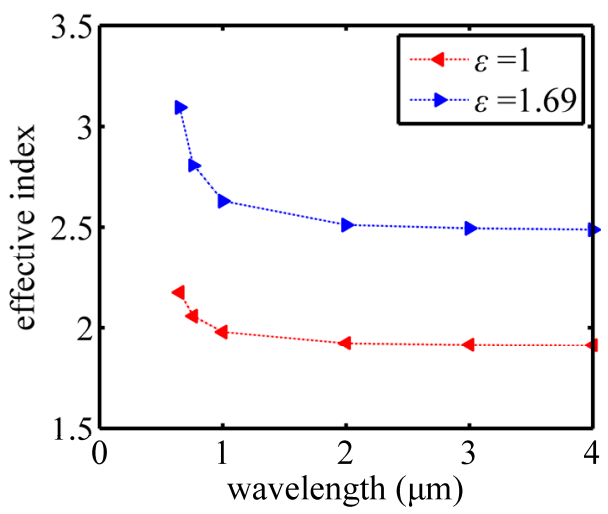

Figure 7. Effective indices of the central waveguide filled with a dielectric of $\varepsilon=1$ and the side waveguide filled with a dielectric of $\varepsilon=1.69$ for various wavelengths.

\section{Conclusions}

In summary, based on Hamiltonian optics, a metallic graded-index (MGRIN) lens composed of an array of coupled metallic waveguides is proposed to focus light at the nanoscale. In contrast to the design of a conventional GRIN lens, the effective index for an MGRIN lens increases from the center to the side due to the negative refraction effect of coupled metallic waveguide arrays. The focusing 
behavior and performance of an MGRIN lens are investigated in detail via the 2D FDTD simulation method, including focal length, focal depth, and field intensity. Numerical results demonstrate that ultra-deep sub-diffraction-limit focusing $(\lambda / 500)$ with a highly enhanced field intensity and long focal depth can be achieved by an MGRIN lens. Moreover, this structure has the ability to focus over a broad range of wavelengths from visible to infrared. Such nanofocusing for broadband wavelengths could hardly be realized by previously reported methods. More importantly, the confinement capability of light based on our design can be further enhanced, provided that the metallic waveguide width can decrease further. The presented MGRIN lens shows great potential for applications in wavelength division multiplexing, nanolithography, and highly integrated optical circuits.

Acknowledgments: The authors acknowledge the financial support from the National Natural Science Foundation of China (Grant Nos. 51375400, 51622509), the Specific Project for the National Excellent Doctorial Dissertations (201430), the 111 Project (Grant No. B13044), and the Innovation Foundation for Doctor Dissertation of Northwestern Polytechnical University (CX201606).

Author Contributions: The manuscript was written through contributions of all authors. All authors have given approval to the final version of this manuscript.

Conflicts of Interest: The authors declare no conflict of interest.

\section{References}

1. Abbe, E. Resolution of microscopes. Arch. Mikrosk. Anat. 1873, 9, 413-425. [CrossRef]

2. Zhang, X.; Liu, Z. Superlenses to overcome the diffraction limit. Nat. Mater. 2008, 7, 435-441. [CrossRef] [PubMed]

3. Gramotnev, D.K.; Bozhevolnyi, S.I. Plasmonics beyond the diffraction limit. Nat. Photon. 2010, 4, 83-91. [CrossRef]

4. Barnes, W.L.; Dereux, A.; Ebbesen, T.W. Surface plasmon subwavelength optics. Nature 2003, 424, 824-830. [CrossRef] [PubMed]

5. Fang, N. Sub-Diffraction-Limited Optical Imaging with a Silver Superlens. Science 2005, 308, $534-537$. [CrossRef] [PubMed]

6. Schuller, J.A.; Barnard, E.S.; Cai, W.; Jun, Y.C.; White, J.S.; Brongersma, M.L. Plasmonics for extreme light concentration and manipulation. Nat. Mater. 2010, 9, 193-204. [CrossRef] [PubMed]

7. Han, Z.; Bozhevolnyi, S.I. Radiation guiding with surface plasmon polaritons. Rep. Prog. Phys. 2013, 76, 16402. [CrossRef] [PubMed]

8. Gramotnev, D.K.; Bozhevolnyi, S.I. Nanofocusing of electromagnetic radiation. Nat. Photon. 2014, 8, 13-22. [CrossRef]

9. Novotny, L.; Hulst, N.V. Antennas for light. Nat. Photon. 2011, 5, 83-90. [CrossRef]

10. Babadjanyan, A.J.; Margaryan, N.L.; Nerkararyan, K.V. Superfocusing of surface polaritons in the conical structure. J. Appl. Phys. 2000, 87, 3785-3788. [CrossRef]

11. Stockman, M.I. Nanofocusing of optical energy in tapered plasmonic waveguides. Phys. Rev. Lett. 2004, 93, 137404. [CrossRef] [PubMed]

12. Issa, N.A.; Guckenberger, R. Optical nanofocusing on tapered metallic waveguides. Plasmon. 2007, 2, 31-37. [CrossRef]

13. Ropers, C.; Neacsu, C.C.; Elsaesser, T.; Albrecht, M.; Raschke, M.B.; Lienau, C. Grating-Coupling of Surface Plasmons onto Metallic Tips: A Nanoconfined Light Source. Nano Lett. 2007, 7, 2784-2788. [CrossRef] [PubMed]

14. Gramotnev, D.K.; Vogel, M.W. Ultimate capabilities of sharp metal tips for plasmon nanofocusing, near-field trapping and sensing. Phys. Lett. A 2011, 375, 3464-3468. [CrossRef]

15. Kravtsov, V.; Ulbricht, R.; Atkin, J.M.; Raschke, M.B. Plasmonic nanofocused four-wave mixing for femtosecond near-field imaging. Nat. Nanotechnol. 2016, 11, 459-464. [CrossRef] [PubMed]

16. Nerkararyan, K.V. Superfocusing of a surface polariton in a wedge-like structure. Phys. Rev. A 1997, 237, 103-105. [CrossRef]

17. Durach, M.; Rusina, A.; Stockman, M.I.; Nelson, K. Toward Full Spatiotemporal Control on the Nanoscale. Nano Lett. 2007, 7, 3145-3149. [CrossRef] [PubMed]

18. Vernon, K.C.; Gramotnev, D.K.; Pile, D.F.P. Adiabatic nanofocusing of plasmons by a sharp metal wedge on a dielectric substrate. J. Appl. Phys. 2007, 101, 104312. [CrossRef]

19. Verhagen, E.; Polman, A.; Kuipers, L.K. Nanofocusing in laterally tapered plasmonic waveguides. Opt. Express 2008, 16, 45-57. [CrossRef] [PubMed] 
20. Verhagen, E.; Spasenović, M.; Polman, A.; Kuipers, L.K. Nanowire plasmon excitation by adiabatic mode transformation. Phys. Rev. Lett. 2009, 102, 203904. [CrossRef] [PubMed]

21. Umakoshi, T.; Saito, Y.; Verma, P. Highly efficient plasmonic tip design for plasmon nanofocusing in near-field optical microscopy. Nanoscale 2016, 8, 5564-5634. [CrossRef] [PubMed]

22. Pile, D.F.P.; Gramotnev, D.K. Adiabatic and nonadiabatic nanofocusing of plasmons by tapered gap plasmon waveguides. Appl. Phys. Lett. 2006, 89, 41111. [CrossRef]

23. Ginzburg, P.; Arbel, D.; Orenstein, M. Gap plasmon polariton structure for very efficient microscale-to-nanoscale interfacing. Opt. Lett. 2006, 31, 3288-3290. [CrossRef] [PubMed]

24. Gramotnev, D.K.; Pile, D.F.; Vogel, M.W.; Zhang, X. Local electric field enhancement during nanofocusing of plasmons by a tapered gap. Phys. Rev. B 2007, 75, 035431. [CrossRef]

25. Vedantam, S.; Lee, H.; Tang, J.; Conway, J.; Staffaroni, M.; Yablonovitch, E. A Plasmonic Dimple Lens for Nanoscale Focusing of Light. Nano Lett. 2009, 9, 3447-3452. [CrossRef] [PubMed]

26. Choo, H.; Kim, M.; Staffaroni, M.; Seok, T.J.; Bokor, J.; Cabrini, S.; Schuck, P.J.; Wu, M.C.; Yablonovitch, E. Nanofocusing in a metal-insulator-metal gap plasmon waveguide with a three-dimensional linear taper. Nat. Photon. 2012, 6, 838-844. [CrossRef]

27. Volkov, V.S.; Bozhevolnyi, S.I.; Rodrigo, S.G.; Martín-Moreno, L.; García-Vidal, F.J.; Devaux, E.; Ebbesen, T.W. Nanofocusing with Channel Plasmon Polaritons. Nano Lett. 2009, 9, 1278-1282. [CrossRef] [PubMed]

28. Sorger, V.J.; Oulton, R.F.; Ma, R.M.; Zhang, X. Toward integrated plasmonic circuits. MRS Bull. 2012, 37, 728-738. [CrossRef]

29. Chung, T.; Lee, S.Y.; Song, E.Y.; Chun, H.; Lee, B. Plasmonic nanostructures for nano-scale bio-sensing. Sensors 2011, 11, 10907-10929. [CrossRef] [PubMed]

30. Frey, H.; Witt, S.; Felderer, K.; Guckenberger, R. Highresolution imaging of single fluorescent molecules with the optical nearfield of a metal tip. Phys. Rev. Lett. 2004, 93, 200801. [CrossRef] [PubMed]

31. Juan, M.L.; Righini, M.; Quidant, R. Plasmon nano-optical tweezers. Nat. Photon. 2011, 5, 349-356. [CrossRef]

32. Economou, E.N. Surface plasmons in thin films. Phys. Rev. 1969, 182, 539. [CrossRef]

33. Xu, T.; Du, C.; Wang, C.; Luo, X. Subwavelength imaging by metallic slab lens with nanoslits. Appl. Phys. Lett. 2007, 91, 201501. [CrossRef]

34. Verslegers, L.; Catrysse, P.B.; Yu, Z.; Fan, S. Planar metallic nanoscale slit lenses for angle compensation. Appl. Phys. Lett. 2009, 95, 071112. [CrossRef]

35. Verslegers, L.; Catrysse, P.B.; Yu, Z.; White, J.S.; Barnard, E.S.; Brongersma, M.L.; Fan, S. Planar lenses based on nanoscale slit arrays in a metallic film. Nano Lett. 2009, 9, 235-238. [CrossRef] [PubMed]

36. Chen, Q.; Cumming, D.R. Visible light focusing demonstrated by plasmonic lenses based on nano-slits in an aluminum film. Opt. Express 2010, 18, 14788-14793. [CrossRef] [PubMed]

37. Zhu, Y.; Yuan, W.; Yu, Y.; Diao, J. Metallic planar lens formed by coupled width-variable nanoslits for superfocusing. Opt. Express 2015, 23, 20124-20131. [CrossRef] [PubMed]

38. Gordon, R. Proposal for superfocusing at visible wavelengths using radiationless interference of a plasmonic array. Phys. Rev. Lett. 2009, 102, 207402. [CrossRef] [PubMed]

39. Verslegers, L.; Catrysse, P.B.; Yu, Z.; Fan, S. Deep-subwavelength focusing and steering of light in an aperiodic metallic waveguide array. Phys. Rev. Lett. 2009, 103, 033902. [CrossRef] [PubMed]

40. Yariv, A.; Yeh, O. Photonics: Optical Electronics in Modern Communications; Oxford Univercity Press: New York, NY, USA, 2006.

41. Reino, C.G.; Pérez, M.V.; Bao, C. Gradient-Index Optics: Fundamentals and Applicatioins; Springer: New York, NY, USA, 2002.

42. Fan, X.; Wang, G.P.; Lee, J.C.W.; Chan, C.T. All-angle broadband negative refraction of metal waveguide arrays in the visible range: theoretical analysis and numerical demonstration. Phys. Rev. Lett. 2006, 97, 073901. [CrossRef] [PubMed]

43. Babar, S.; Weaver, J.H. Optical constants of Cu, Ag, and Au revisited. Appl. Opt. 2015, 54, 477-481. [CrossRef]

44. Ferrell, R.A. Characteristic energy loss of electrons passing through metal foils. ii. Dispersion relation and short wavelength cutoff for plasma oscillations. Phys. Rev. 1956, 107, 450-462. [CrossRef]

(C) 2017 by the authors. Licensee MDPI, Basel, Switzerland. This article is an open access article distributed under the terms and conditions of the Creative Commons Attribution (CC BY) license (http://creativecommons.org/licenses/by/4.0/). 